\title{
Exploiting Regional Differences: A Spatially Adaptive Random Access
}

\author{
Dong Min Kim and Seong-Lyun Kim
}

\begin{abstract}
In this paper, we discuss the potential for improvement of the simple random access scheme by utilizing local information such as the received signal-to-interference-plus-noiseratio (SINR). We propose a spatially adaptive random access (SARA) scheme in which the transmitters in the network utilize different transmit probabilities depending on the local situation. In our proposed scheme, the transmit probability is adaptively updated by the ratio of the received SINR and the target SINR. We investigate the performance of the spatially adaptive random access scheme. For the comparison, we derive an optimal transmit probability of ALOHA random access scheme in which all transmitters use the same transmit probability. We illustrate the performance of the spatially adaptive random access scheme through simulations. We show that the performance of the proposed scheme surpasses that of the optimal ALOHA random access scheme and is comparable with the CSMA/CA scheme.
\end{abstract}

Index Terms-Random access, distributed scheduling, SINRbased interference model, adaptive algorithm.

\section{INTRODUCTION}

\section{A. Brief Description of Spatially Adaptive Random Access} (SARA)

Assume that the nodes in the network are randomly located (sensors in forest, people in crowded area). If a node is located in the relatively dense environment, the transmission of the node would be frequently failed due to the aggregate interference. In this case, the node should lower the transmit probability to resolve the contention. On the other hand, if the node is located in the relatively sparse circumstance, there are a few strong interfering nodes and its transmission may not be interfered. In this case, the node could raise the transmit probability to take advantage of the situation. This observation gives us an intuition to design a new random access scheme.

In this paper, we propose a spatially adaptive random access (SARA) scheme. Each node $i$ behaves as follows:

1) Initialize transmit probability with the largest value, $\phi_{\max }$.

2) Compute the average signal-to-interference-plus-noiseratio (SINR) at time $t, \Gamma_{k(i)}(t)$, during period $T$ as follows:

$$
\Gamma_{k(i)}(t) \approx \frac{1}{T} \sum_{1}^{T}\left[\frac{G_{i, k(i)} P_{i}}{\sum_{u \in \mathcal{T}_{i}^{\prime}} G_{u, k(i)} P_{u}}\right]
$$

Dong Min Kim is with the Department of Electronic Systems, Aalborg University, Fredrik Bajers Vej 7, 9220 Aalborg, Denmark (email: dmk@es.aau.dk).

Seong-Lyun Kim is with the School of Electrical and Electronic Engineering, Yonsei University, 50 Yonsei-Ro, Seodaemun-Gu, Seoul, 120-749, Korea (email: slkim@ramo.yonsei.ac.kr). where the notation $k(i)$ denotes the receiver associated with transmitter $i$. The notation $G_{i, j}$ denotes the channel gain from node $i$ to node $j$. The term $P_{i}$ represents the transmit power of transmitter $i$. The term $\mathcal{T}_{i}^{\prime}$ denotes the subset of the concurrent transmission nodes when node $i$ transmits.

3) Update transmit probability $\phi_{i}$ as follows:

$$
\phi_{i}(t+1)=\min \left\{\max \left\{\phi_{\min }, \frac{\Gamma_{k(i)}(t)}{\beta}\right\}, \phi_{\max }\right\},
$$

where the notations $\phi_{\min }$ and $\phi_{\max }$ represent minimum and maximum values of the transmit probability, respectively. The notation $\beta$ denotes a target SINR threshold.

SARA is a variant of ALOHA, where each transmitter updates the transmit probability depending on the local situation. We verify the convergence property using the standard interference function approach ([1], [2]) and simulations. SARA improves the average received SINR with a little message passing in the network. Our simulation results show that, for the whole cases we considered, the area spectral efficiency performance of SARA is even better than a carrier sense multiple access with collision avoidance (CSMA/CA), where the carrier sensing range is set by doubling the transmission distance as a conventional setting.

\section{B. Motivation and Related Works}

The ALOHA protocol [3] is the most well-known distributed random access scheme. The transmit probability controls the operation of the ALOHA protocol. In [4], the authors derive an optimal transmit probability under the protocol model, where the transmission fails if two or more nodes are transmitting simultaneously. To improve the performance of an ALOHA network, researchers conducted several studies using a simple protocol model to achieve proportional fairness and max-min fairness [5]-[7]. In [8]-[10], the authors investigated optimal random access approaches achieving network utility maximization using a family of $\alpha$-fair utility functions [11] in the protocol model. However, due to the characteristics of the wireless channel [12], the receiver may successfully receive the signal if the concurrent transmitters are far away. The physical model [13] considers the effect of such accumulated multi-user interference.

In practice, interfering nodes are randomly located. In this regard, stochastic geometry [14]-[22] is a useful mathematical tool to model such randomness. In [23], the authors provide a stochastic geometry-based analytical framework of ALOHA. 


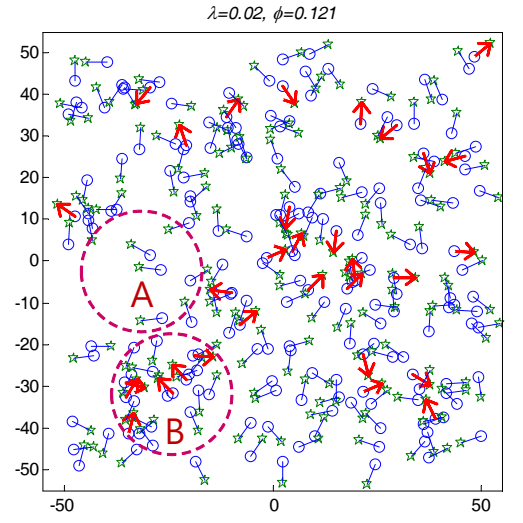

Fig. 1. A snapshot of network topology. The small circles represent transmitters, and the connected pentagrams represent associated receivers. The arrows represent active communication pairs. The node density, $\lambda$, is 0.02 and the transmit probability, $\phi$, is 0.121 . The nodes in subarea A are located in a relatively sparse environment. On the other hand, the nodes in subarea B are located in a relatively dense environment.

In a recent study [24], the authors investigated an adaptive ALOHA using a SINR model from the stochastic geometry point of view. The authors of [24] focus on achieving proportional fairness while we concentrate on improving the area spectral efficiency. In [25], the authors investigate the SINR-based random access protocol. Later, in [26], the authors propose an adaptive interference pricing scheme to find a local optimal solution of the network utility maximization problem. They adopted a game theoretic framework ([27], [28]) to analyze multiple access control (MAC). The proposed approaches in [25] and [26] require a large number of message exchanges among the transmitters to inform their transmit probabilities to the others.

CSMA/CA is more advanced than ALOHA in that it has the ability to adapt the local situation through carrier sensing. The conventional ALOHA-like random access cannot behave adaptively because the transmit probability is fixed by a single optimal value. The optimal values of transmit probabilities are different in dense and sparse environments, and all nodes in the network should not have the same transmit probability.

Let us assume that the nodes are deployed as shown in Figure 1. About 200 communication pairs are randomly distributed in a rectangular area. In this case, the node density is 0.02 , and all transmitters have a fixed transmission probability. The nodes in subarea A are located in a relatively sparse environment; their transmissions may not be interfered. On the other hand, the nodes in subarea B are located in a relatively dense environment, and the transmissions of the nodes in B would frequently fail due to heavy interference. The nodes in A may want to utilize a relatively high transmit probability, and in B, a low probability. To improve the performance of such an ALOHA-like random access scheme, we devise SARA, which adjusts the transmit probability according to the local circumstance.

The main contributions of this paper are summarized as follows:
TABLE I

KeY MATHEMATICAL NOTATIONS.

\begin{tabular}{|c|c|}
\hline$\lambda$ & Node density \\
\hline $\mathcal{A}$ & Area of interesting region \\
\hline$k(i)$ & Associated receiver of transmitter $i$ \\
\hline$r_{t}$ & Communication distance \\
\hline$P_{i}$ & Emission power of transmitter $i$ \\
\hline$\phi_{i}$ & Transmit probability of transmitter $i$ \\
\hline$\phi_{\min } / \phi_{\max }$ & Minimum/maximum transmit probability \\
\hline$G_{i, j}$ & Channel gain from node $i$ to node $j$ \\
\hline$\alpha$ & Path loss exponent \\
\hline$\gamma_{\mathcal{T}_{i}^{j}}$ & Instantaneous SINR of receiver $k(i)$ \\
\hline $\mathcal{T}_{i}$ & Superset of interfering nodes when node $i$ transmits \\
\hline $\mathcal{T}_{i}^{\prime}$ & Subset of interfering nodes when node $i$ transmits \\
\hline $\mathcal{T}_{i}^{J}$ & $j$-th subset of interfering nodes when node $i$ transmits \\
\hline$\beta$ & Target SINR threshold \\
\hline$r_{i}$ & Data rate of transmitter $i$ \\
\hline$\eta$ & Area spectral efficiency \\
\hline$p_{s}$ & Success probability \\
\hline$\phi^{*}$ & Optimal transmit probability \\
\hline $\mathcal{N}$ & Set of transmitters \\
\hline$\Gamma_{k(i)}$ & Average SINR \\
\hline $\mathbf{\Phi}$ & Vector of all transmit probabilities \\
\hline $\mathbf{\Phi}_{-i}$ & Vector of all transmission probabilities except node $i$ \\
\hline
\end{tabular}

- We proposed a distributed SARA scheme, where the average received SINR is improved with a little message passing with other nodes in the network.

- We present the convergence property of the proposed scheme using the standard interference function method and simulations.

- We show the area spectral efficiency performance of the proposed scheme is better than that of ALOHA and comparable with CSMA/CA by simulations.

The rest of the paper is organized as follows. In Section II, we describe the system model. In Section III, we investigate our SARA scheme. In Section IV, we analyze the convergence property of the proposed scheme. We verify the performance through simulations in Section V. Section VI concludes the paper.

\section{System Model}

As shown in Figure 11, a random wireless network of a single radio channel is considered, where each transmitter, $i$, is associated with a receiver, $k(i)$, over a shared wireless channel. The transmitters/receivers are randomly scattered in the network. Each transmitter always has ample data to send. We assume that the time is slotted and synchronized so that transmissions begin with a time slot and continue during the slot length. The transmitter/receiver pair can be changed over the time. However, we focus on a snapshot of the overall communication process, where the network topology is fixed during each slot.

The transmitter $i$ attempts to send its data with transmit probability $\phi_{i}$. The channel gain from node $i$ to node $j$, $G_{i, j}$, depends on the distance between the transmitter and the receiver with path loss exponent $\alpha$ and Rayleigh fading. The stochastic process of the wireless channel is ergodic. With a single shared channel, the concurrent transmissions cause cochannel interference. The instantaneous SINR of receiver 
$k(i), \gamma_{\mathcal{T}_{i}^{\prime}}$, is given by

$$
\gamma_{\mathcal{T}_{i}^{\prime}}=\frac{G_{i, k(i)} P_{i}}{\sum_{u \in \mathcal{T}_{i}^{\prime}} G_{u, k(i)} P_{u}+W_{k(i)}}, \quad \mathcal{T}_{i}^{\prime} \in \mathcal{T}_{i},
$$

where we consider the interference limited network. Then, the noise power term $W_{k(i)}$ is omitted from Eq. (2) and we deal with the signal-to-interference-ratio (SIR). The notation $\mathcal{T}_{i}$ denotes the superset of concurrent transmission nodes (interfering nodes) when node $i$ transmits. When there are $n$ transmitters in the networks, the cardinality of $\mathcal{T}_{i}$ is $2^{n-1}$. The notation $\mathcal{T}_{i}^{\prime}$ denotes the subset of the simultaneously transmitting nodes when node $i$ transmits. Similarly, the notation $\mathcal{T}_{i}^{j}$ denotes the $j$-th subset of the simultaneously transmitting nodes when node $i$ transmits. For example, assume that the network consists of three pairs $\{1,2,3\}$. If transmitter 1 is active, the superset of concurrent transmitting nodes, $\mathcal{T}_{1}$, is $\{\{\},\{2\},\{3\},\{2,3\}\}$, and $\mathcal{T}_{1}^{1}=\{\}, \mathcal{T}_{1}^{2}=\{2\}, \mathcal{T}_{1}^{3}=\{3\}, \mathcal{T}_{1}^{4}=\{2$, $3\}$. We also use the notation $\gamma_{k(i)}$ to represent the instantaneous SINR of receiver $k(i)$ when there is no need to specify the subset of the simultaneously transmitting nodes.

We assume that the transmitter utilizes a fixed and robust coding/modulation scheme that achieves the Shannon capacity. Then, there exists a minimum SINR threshold to successfully decode the received signal at the receiver. For a given target SINR threshold $\beta$, transmission is successful if $\gamma_{\mathcal{T}_{i}^{\prime}} \geq \beta$ is satisfied and the data rate of each transmission is $r_{i}=\log _{2}(1+\beta)$, where we assume a unit bandwidth.

\section{Spatially Adaptive Random Access}

\section{A. Improving Area Spectral Efficiency}

The area spectral efficiency (ASE) $\eta$ is the sum of data rates per unit bandwidth in the unit area ([29], [30]). To focus on a network-wide performance, we use $\eta$ as a performance metric. To maximize $\eta$, we formulate an optimization problem as follows:

$$
\begin{array}{ll}
\max & \eta=\log _{2}(1+\beta) \sum_{i} \mathbb{E}\left[\mathbf{1}_{\gamma_{k(i)} \geq \beta}\right] \\
\text { s.t. } & \phi_{\min } \leq \phi_{i} \leq \phi_{\max }, \quad \forall i,
\end{array}
$$

where $\mathbf{1}_{\gamma_{k(i)} \geq \beta}$ denotes the indicator function defined as 1 if $\gamma_{k(i)} \geq \beta$, otherwise 0 . The term $\sum_{i} \mathbb{E}\left[\mathbf{1}_{\gamma_{k(i)} \geq \beta}\right]$ represents the expected value of the number of successfully transmitting nodes in the unit area. The term $\gamma_{k(i)}$ is a random variable of the instantaneous SINR of the receiver $k(i)$. The term $\sum_{i} \mathbb{E}\left[\mathbf{1}_{\gamma_{k(i)} \geq \beta}\right]$ is a function of $\phi_{i}$ 's as follows:

$$
\sum_{i} \mathbb{E}\left[\mathbf{1}_{\gamma_{k(i)} \geq \beta}\right]=\sum_{i}\left(\sum_{j=1}^{2^{n-1}}\left(\prod_{l \in \mathcal{T}_{i}^{j}} \phi_{l}\right)\left(\prod_{m \in \mathcal{N} \backslash\left\{\mathcal{T}_{i}^{j}, i\right\}}\left(1-\phi_{m}\right)\right) \mathbf{1}_{\gamma_{\mathcal{T}_{i}^{j}} \geq \beta}\right),
$$

where $\mathcal{N}$ is a set of all transmitters. The detailed derivation is in Appendix A. If $\beta$ is constant, maximizing (3) is equal to maximize (4). To maximize Eq. (4), we should find all $\gamma_{\mathcal{T}_{i}^{j}}$ 's. This means that we should compute all combinations of interferers. This is a combinatorial optimization problem which becomes harder to solve as the number of nodes in the network increases.

\section{B. Utility Maximization Problem}

In an effort to solve Eq. (3) in a decentralized manner, we first define the utility function of node $i$ using (4). Let us define the function $f_{i}\left(\boldsymbol{\Phi}_{-i}\right)$ as follows:

$$
f_{i}\left(\boldsymbol{\Phi}_{-i}\right)=\sum_{j=1}^{2^{n-1}}\left(\prod_{l \in \mathcal{T}_{i}^{j}} \phi_{l}\right)\left(\prod_{m \in \mathcal{N} \backslash\left\{\mathcal{T}_{i}^{j}, i\right\}}\left(1-\phi_{m}\right)\right) \mathbf{1}_{{\mathcal{T}_{i}^{j}}^{j} \geq \beta},
$$

where $\boldsymbol{\Phi}_{-i}$ is a vector of the transmit probabilities of all nodes except node $i$. The function $f_{i}\left(\boldsymbol{\Phi}_{-i}\right)$ is the expression for outer summation in (4). Eq. (4) can be expressed as follows and we define it as the utility function of the node $i$ :

$$
U_{i}\left(\phi_{i}\right)=f_{i}\left(\boldsymbol{\Phi}_{-i}\right)+\sum_{w \neq i} f_{w}\left(\boldsymbol{\Phi}_{-w}\right)
$$

Then each communication pair solves the following utility maximization problem:

$$
\begin{array}{ll}
\max & U_{i}\left(\phi_{i}\right) \\
\text { s.t. } & \phi_{\min } \leq \phi_{i} \leq \phi_{\max } .
\end{array}
$$

By inspection, we found that the utility function can be expressed as follows:

$$
U_{i}\left(\phi_{i}\right)=R_{i} \phi_{i}-C_{i} \phi_{i}+o,
$$

where $R_{i}$ is interpreted as the reward for the action transmitting with probability $\phi_{i}$ and $C_{i}$ is interpreted as the cost for the action transmitting with probability $\phi_{i}$. The term $o$ represents all irrelevant expressions with control variable $\phi_{i}$. For example, assuming three communication pairs are in the network. The utility function for communication pair 1 is as follows:

$$
U_{1}\left(\phi_{1}\right)=f_{1}\left(\boldsymbol{\Phi}_{-1}\right)+f_{2}\left(\boldsymbol{\Phi}_{-2}\right)+f_{3}\left(\boldsymbol{\Phi}_{-3}\right) .
$$

Above (9) can be expressed as

$$
\begin{array}{r}
U_{1}\left(\phi_{1}\right)=\left(\phi_{3} \mathbf{1}_{\gamma_{2,\{1,3\}} \geq \beta}+\left(1-\phi_{3}\right) \mathbf{1}_{\gamma_{2,\{1\}} \geq \beta}+\phi_{2} \mathbf{1}_{\gamma_{3,\{1,2\}} \geq \beta}+\left(1-\phi_{2}\right) \mathbf{1}_{\gamma_{3,\{1\}} \geq \beta}\right) \phi_{1} \\
-\left(\phi_{3} \mathbf{1}_{\gamma_{2,\{3\}} \geq \beta}+\left(1-\phi_{3}\right) \mathbf{1}_{\gamma_{2,\{\}} \geq \beta}+\phi_{2} \mathbf{1}_{\gamma_{3,\{1\}} \geq \beta}+\left(1-\phi_{2}\right) \mathbf{1}_{\gamma_{3,\{\}} \geq \beta}\right) \phi_{1}+o .
\end{array}
$$

The problem (3) can be solved by gathering the solution of (7). We still should know all $\gamma_{\mathcal{T}_{i}^{j}}$ 's for each node $i$. As we mentioned in Section II, the number of combinations is $2^{n-1}$. The problems (3) and (7) have to choose an optimal simultaneous transmission set for every transmission instantaneously. This is not a practical scenario, especially with a large number of nodes. A feasible and possible way is handling the average performance, not the instantaneous performance. In this regard, we approximate $U_{i}\left(\phi_{i}\right)$ as a utility function of node $i$ as follows 1

$$
U_{i}\left(\phi_{i}\right) \approx\left[\frac{1}{\beta}\left(\sum_{j=1}^{2^{n-1}}\left(\prod_{l \in \mathcal{T}_{i}^{j}} \phi_{l}\right)\left(\prod_{m \in \mathcal{N} \backslash\left\{\mathcal{T}_{i}^{j}, i\right\}}\left(1-\phi_{m}\right)\right) \gamma_{\mathcal{T}_{i}^{j}}\right)\right]_{\phi_{\min }}^{\phi_{\max }} \phi_{i}-\frac{1}{2} \phi_{i}^{2} .
$$

Eq. (10) retains reward and cost structure of (8) and changes actual reward and cost expressions to obtain readily. Therefore the solution of optimization problem with (10) is not an exact solution of (3) and (7), but an approximated one. The node $i$,

\footnotetext{
${ }^{1}$ The notation $[\cdot]_{a}^{b}$ denotes $\min (\max (a, \cdot), b)$.
} 
who transmits with the probability $\phi_{i}$, obtains the reward as a form of the ratio of the average SINR to the target SINR. As we achieve higher average SINR, the reward increases. The bad effect on the network (increasing contention and interference) is assessed as the cost part of (10). Our utility function has a property to penalize the occurrence of the interference. It makes sure that the radio spectrum resources are efficiently shared.

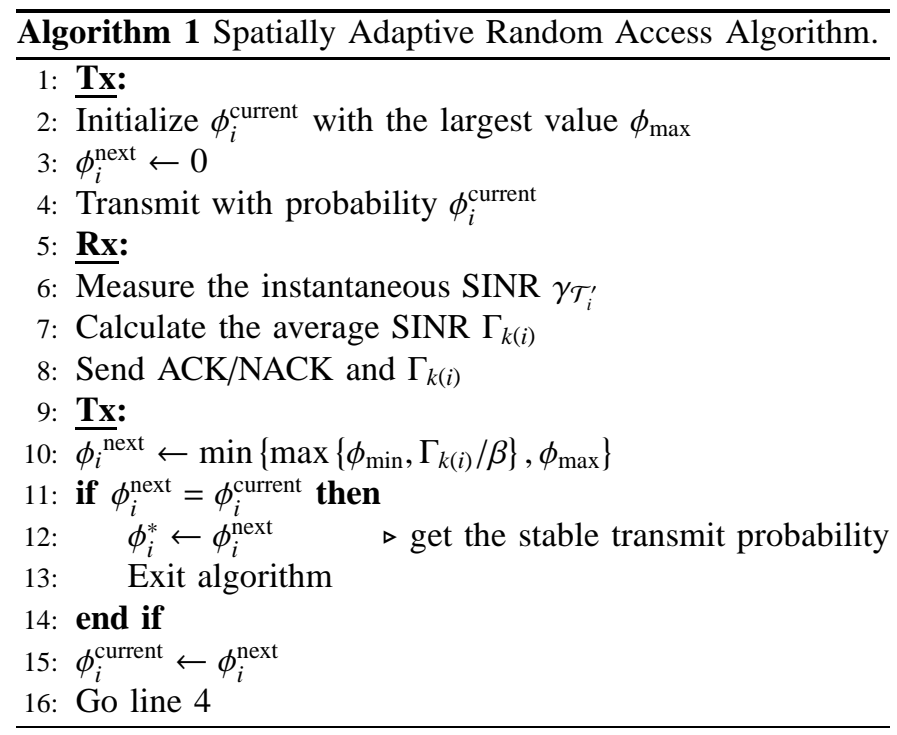

The problem (7) is the one dimensional convex optimization because the second derivative of (10) is $\frac{\partial^{2} U_{i}(\boldsymbol{\Phi})}{\partial \phi_{i}^{2}}=-1$ and the constraint set is convex. Therefore the solution occurs $\frac{\partial U_{i}(\boldsymbol{\Phi})}{\partial \phi_{i}}=$ 0 or the boundary of the constraint set as follows: 2

$$
\frac{\partial U_{i}(\boldsymbol{\Phi})}{\partial \phi_{i}}=\left[\frac{1}{\beta}\left(\sum_{j=1}^{2^{n-1}}\left(\prod_{l \in \mathcal{T}_{i}^{j}} \phi_{l}\right)\left(\prod_{m \in \mathcal{N} \backslash\left\{\mathcal{T}_{i}^{j}, i\right\}}\left(1-\phi_{m}\right)\right) \gamma_{\mathcal{T}_{i}^{j}}\right)\right]_{\phi_{\min }}^{\phi_{\max }}-\phi_{i}=0 .
$$

This yields a theoretic form of an iterative algorithm as follows:

$$
\phi_{i}(t+1)=\left[\frac{1}{\beta}\left(\sum_{j=1}^{2^{n-1}}\left(\prod_{l \in \mathcal{T}_{i}^{j}} \phi_{l}(t)\right)\left(\prod_{m \in \mathcal{N} \backslash\left\{\mathcal{T}_{i}^{j}, i\right\}}\left(1-\phi_{m}(t)\right)\right) \gamma_{\mathcal{T}_{i}^{j}}\right)\right]_{\phi_{\min }}^{\phi_{\max }} .
$$

To obtain the exact value of $\gamma_{\mathcal{T}_{i}^{j}}$, the nodes in the network need to frequently exchange the message with neighbor nodes to acquire the transmit probabilities of all other transmitters. To reduce this complexity, we use the time-averaged SINR update the transmit probability as follows:

$$
\Gamma_{k(i)}=\mathbb{E}\left[\frac{G_{i, k(i)} P_{i}}{\sum_{u \in \mathcal{T}_{i}^{\prime}} G_{u, k(i)} P_{u}}\right] \approx \frac{1}{T} \sum_{1}^{T}\left[\frac{G_{i, k(i)} P_{i}}{\sum_{u \in \mathcal{T}_{i}^{\prime}} G_{u, k(i)} P_{u}}\right] .
$$

In our system, the success of a transmission is determined by an instantaneous SINR and the target SINR $\beta$. The instantaneous SINR changes with a small-time-scale (milliseconds) due to the Rayleigh fading, which is independent of a spatial

\footnotetext{
${ }^{2}$ Our utility function is designed that the gradient at the optimal point is always zero. See Appendix B.
}

random distribution of nodes in the network. To get rid of the effect of fading and to reflect the distribution of nodes, we utilize the average SINR, which varies with a largetime-scale (seconds). The average is measured by each of the nodes during the buffered period $T$. Even though the ensemble average is more accurate than the time average, the time average with a sufficient period can approximate the ensemble average when the wireless channel is ergodic. In this regard, the time-averaged SINR value is an indicator of the network condition. If the average SINR is lower than the target SINR, there could be many transmitters contending for the opportunity to transmit.

Our algorithm, shown in Algorithm 1 and also briefly described in Section I-A, finds the transmit probabilities maximizing the utility function, Eq. (10). The average SINR computation is done by the receiver. To calculate the SINR, the receiver measures the received signal strength (RSS). The receiver does not require explicit information about transmit power and path loss of other users. To inform the success/failure of transmission, the receiver sends out the acknowledgement signal (ACK/NACK) for each transmission. The receiver should notify its transmitter of the average SINR when the receiver transmits the acknowledgement signal (piggybacking).

The transmit probability is updated by the ratio of the average SINR to the target SINR threshold. If the average SINR is larger than the target $\beta$, the network situation is favorable for that communication pair. The pair may be isolated from the others. Therefore it is highly probable that the transmission of this transmitter will not interfere with the communications of the others. To promote more chances to transmit, the transmit probability is set to the maximum. On the other hand, as the average SINR is getting lower, the communication pair experiences more contending situation. The transmit probability should be lowered to resolve the contention by means of the ratio of the average SINR to the target SINR. The convergence property of the proposed algorithm is given in the next section.

\section{Convergence Property of SARA}

In this section, the convergence property of SARA is verified using the standard interference function method ([1], [2]). A standard interference function $I(\boldsymbol{\Phi})$ has following properties:

1) Positivity: $I(\boldsymbol{\Phi})>0$,

2) Monotonicity: $\boldsymbol{\Phi} \geq \boldsymbol{\Phi}^{\prime} \Rightarrow I(\boldsymbol{\Phi}) \geq I\left(\boldsymbol{\Phi}^{\prime}\right)$,

3) Scalability: $\forall \alpha>1, \quad \alpha I(\boldsymbol{\Phi}) \geq I(\alpha \boldsymbol{\Phi})$.

The iterative algorithm using the standard interference function $\boldsymbol{\Phi}(t+1)=I(\boldsymbol{\Phi}(t))$ converges to a fixed point [1]. The authors of [2] extend the framework of [1] using a novel class of iterative functions. They define the two-sided scalability:

$$
\forall \theta>1, \frac{1}{\theta} \boldsymbol{\Phi} \leq \boldsymbol{\Phi}^{\prime} \leq \theta \boldsymbol{\Phi} \Rightarrow \frac{1}{\theta} I(\boldsymbol{\Phi}) \leq I\left(\boldsymbol{\Phi}^{\prime}\right) \leq \theta I(\boldsymbol{\Phi}) .
$$

The iterative algorithm using the function that satisfies the two-sided scalability will converge to the unique fixed point [2]. 
TABLE II

Key SIMULATION PARAMETERS

\begin{tabular}{|l|l|}
\hline Parameter & Value \\
\hline Node density & $0.005-0.06$ \\
Communication distance $r_{t}$ & $5 \mathrm{~m}$ \\
Transmit power & $30 \mathrm{dBm}$ \\
Noise floor & $-70 \mathrm{dBm}$ \\
Carrier sensing range for CSMA/CA & $10 \mathrm{~m}$ \\
Target SINR threshold $\beta$ & $0,3,5 \mathrm{~dB}$ \\
Communication space size & $30 \mathrm{~m} \times 30 \mathrm{~m}, 100 \mathrm{~m} \times 100 \mathrm{~m}$ \\
\hline
\end{tabular}

Let,

$$
I(\boldsymbol{\Phi}(t))=\frac{1}{\beta}\left(\sum_{j=1}^{2^{n-1}}\left(\prod_{l \in \mathcal{T}_{i}^{j}} \phi_{l}(t)\right)\left(\prod_{m \in \mathcal{N} \backslash\left\{\mathcal{T}_{i}^{j}, i\right\}}\left(1-\phi_{m}(t)\right)\right) \gamma_{\mathcal{T}_{i}^{j}}\right) .
$$

Eq. (15) satisfies the two-sided scalability. The detailed derivation is in Appendix C. If $I(\boldsymbol{\Phi})$ is a standard function, then $\min \left\{\max \left\{\phi_{\min }, I(\boldsymbol{\Phi})\right\}, \phi_{\max }\right\}$ is also standard (Proposition 5 in [2]).

Our iterative algorithm (12) utilizes the two-sided scalable standard function. Thus, the iterative update algorithm will converge to a fixed point. In the next section, we evaluate the performance of the proposed random access scheme.

\section{Performance Evaluation}

\section{A. General Setting}

The transmitters are distributed according to a homogeneous Poisson point process (PPP) with intensity $\lambda$. In the finite region (of size $\mathcal{A}$ ), nodes are independent and identically distributed with a uniform distribution in the region with a given average number of nodes $(\lambda \mathcal{A})$. Each associated receiver, $k(i)$, is located at a distance of $r_{t}$ from the transmitter $i$ and the direction is random. The receivers also follow the homogeneous PPP by the displacement theorem [15]. The variable transmit distance can be used; however the fixed distance provides a significant tractability in analysis of optimal transmit probability of conventional ALOHA. Also previous researches [31], [32] noted that the variable distance does not provide the fundamentally different capacity characteristics. The key simulation parameters are listed in Table [I.

\section{B. Average SINR Validation and Convergence Simulation}

To evaluate the accuracy of Eq. (13), we conducted a simulation: As shown in Figure 2, a total of 11 transmitter/receiver pairs are distributed on the $30 \mathrm{~m}$ by $30 \mathrm{~m}$ area. The communication distance between a transmitter/receiver pair is $5 \mathrm{~m}$. The transmit power is $30 \mathrm{dBm}$. The target threshold is $3 \mathrm{~dB}$. Figure 3 shows the exact SINR (ensemble average) in Eq. (23) and the time-averaged SINR in Eq. (13). The timeaveraged SINR can approximate the exact ensemble average. The small differences are caused by the fading characteristics of wireless channel.

Figure 4 shows the updated transmit probabilities. The updated probabilities using the time-averaged SINR (1) (without message passing to other pairs) are almost the same as the updated probabilities using the ensemble-averaged SINR (12) (with frequent message passing to other pairs), as shown in

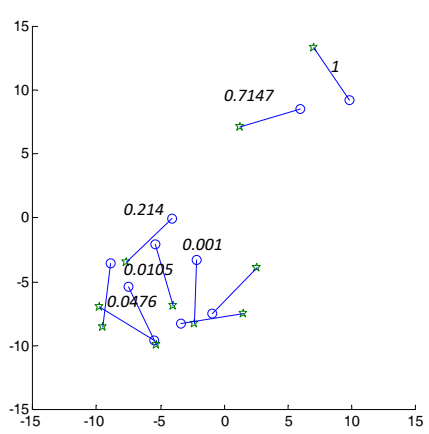

Fig. 2. Spatial setting for SINR validation simulation. Two communication pairs are relatively isolated from the others.

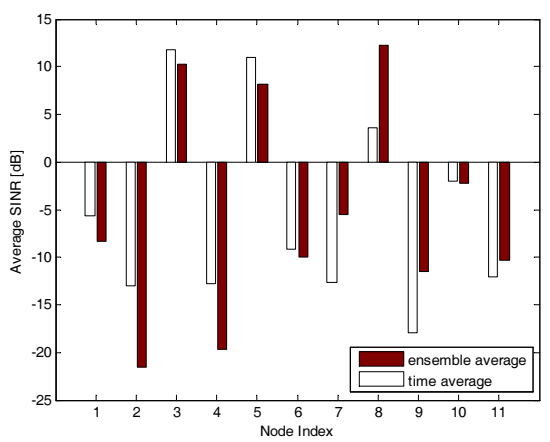

Fig. 3. The average SINR of randomly distributed nodes. The exact SINR is estimated by time averaged SINR.

Figure 4 Using the time-averaged SINR, the transmitter and receiver are only communicating each other. Otherwise, updating probability with the ensemble-averaged SINR requires frequent message exchanging with other communication pairs in order to know their transmit probabilities. Figure 5 shows the time scale dynamics of the transmit probabilities while applying SARA, where the transmit probabilities converges.

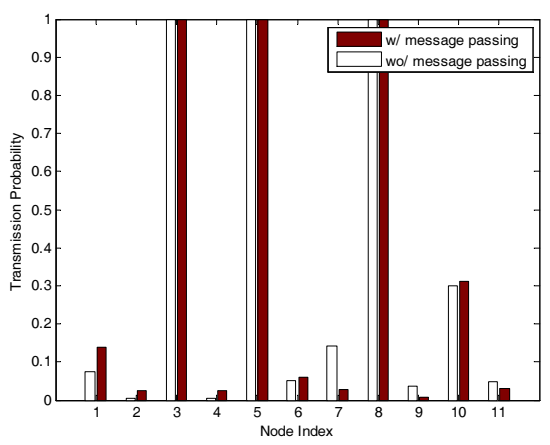

Fig. 4. Comparison of the transmit probabilities using time-averaged SINR (without message passing to other pairs) and using the ensemble-averaged SINR (with frequent message passing to other pairs). 


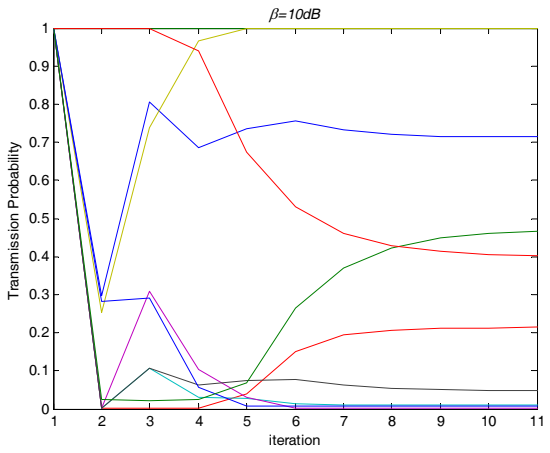

Fig. 5. The trajectory of the transmit probabilities. All transmit probabilities converge to certain values.

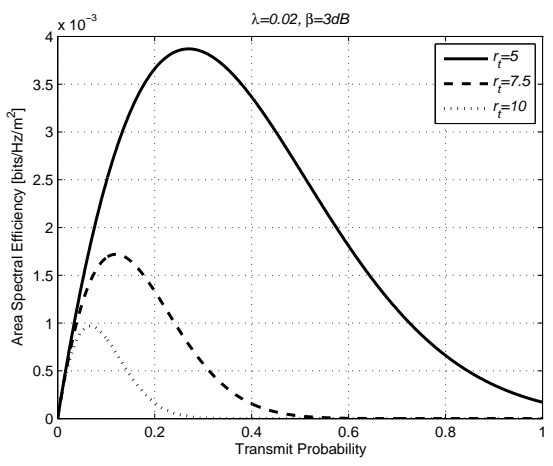

Fig. 6. The area spectral efficiency as a function of transmit probability. The node density, $\lambda$, is 0.02 and the target SINR, $\beta$, is $3 \mathrm{~dB}$.

\section{Optimal Transmit Probability of Conventional ALOHA}

We analyze the performance of the conventional ALOHAlike random access scheme. In this case, all transmitters utilize the same transmit probability $\phi$. In a stochastic geometry point of view, the ASE can be expressed as the product of the successfully transmitting node density and data rate as follows [22]:

$$
\eta=\lambda \phi \log (1+\beta) p_{s}
$$

where the success probability, $p_{s}$, of ALOHA is derived as follows ([33, Proposition 2.1]):

$$
p_{s}=\exp \left(-\lambda \phi r_{t}^{2} \beta^{2 / \alpha} \rho(\alpha)\right),
$$

where $\rho(\alpha)=\frac{2 \pi^{2}}{\alpha} \csc \left(\frac{2 \pi}{\alpha}\right)$. With Eq. (17), we can rewrite the ASE $\eta$ as a function of $\phi$ as follows:

$$
\eta(\phi)=\lambda \phi \log (1+\beta) \exp \left(-\lambda \phi r_{t}^{2} \beta^{2 / \alpha} \rho(\alpha)\right) .
$$

As shown in Figure 6 , there is an optimal $\phi$ that maximizes the ASE of ALOHA Eq. (18):

$$
\phi^{*}=\underset{\phi}{\arg \max } \lambda \phi \log (1+\beta) p_{s} .
$$

The solution of Eq. (19), $\phi^{*}$, is obtained as follows:

$$
\phi^{*}=\frac{1}{\lambda r_{t}^{2} \beta^{2 / \alpha} \rho(\alpha)},
$$

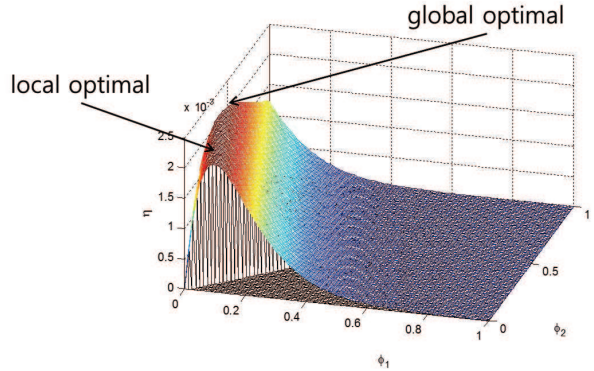

Fig. 7. The area spectral efficiency as a function of transmit probabilities. The node density, $\lambda$, is 0.02 and the target SINR, $\beta$, is $3 \mathrm{~dB}$.

where $\rho(\alpha)=\frac{2 \pi^{2}}{\alpha} \csc \left(\frac{2 \pi}{\alpha}\right)$. The detailed derivation is in Appendix D.

By substituting Eq. (20) into Eq. (18), we have the maximum ASE $\eta^{*}$ of ALOHA as follows:

$$
\eta^{*}=0.3679 \frac{\log (1+\beta)}{r_{t}^{2} \beta^{2 / \alpha} \rho(\alpha)} .
$$

What is interesting in Eq. (21) is that the maximum ASE $\eta^{*}$ of ALOHA is independent of node density $\lambda$. This is because the optimal transmit probability achieving the maximum ASE decreases at the rate of $1 / \lambda$. This scaling characteristic is consistent with that of the protocol model, in which the optimal transmit probability scales with $1 / N$ when there are a total of $N$ transmitters. In the physical model, the effect of target SINR $\beta$ and path-loss exponent $\alpha$ are counted.

Figure 7 shows a more general case, where there are two transmit probabilities $\left(\phi_{1}, \phi_{2}\right)$ in the network. We obtain Eq. (20) when $\phi_{1}=\phi_{2}$. However, the global optimal exists elsewhere. The previous framework cannot improve the performance more than Eq. (20) while there is room for improving. Our approach can improve the performance.

\section{Large-scale Network Simulation}

To quantify the performance of SARA, we conducted a large-scale network simulation. In a $100 \mathrm{~m} \times 100 \mathrm{~m}$ area, various numbers of nodes are distributed according to the node density. The node density varies from 0.005 (sparse case) to 0.06 (dense case). The communication distance is $5 \mathrm{~m}$. The transmission power is $30 \mathrm{dBm}$ and the noise power is $-70 \mathrm{dBm}$.

Figure 8 shows the snapshot of the network topology in the case of $\lambda=0.02$. Even though the same node density is applied, we can observe the regional variance of the population. Figure 8(a) and Figure 8(b) illustrate the dense part of the network. In Figure 8(a), the conventional ALOHA scheme is applied, and the transmitters highly overlap each other. On the other hand, in Figure 8(b) the transmitters are separated by utilizing the SARA scheme. In Figure $8(\mathrm{c})$ and $8(\mathrm{~d})$, the sparse part of the network is depicted. Since the transmit probability of the SARA scheme is adjusted by the number of strong interferers, the transmitters in the sparse situation try to transmit frequently while the transmitters using ALOHA are not.

Figure 9 shows the topology of the active transmitters of the network. In the case of the conventional ALOHA 

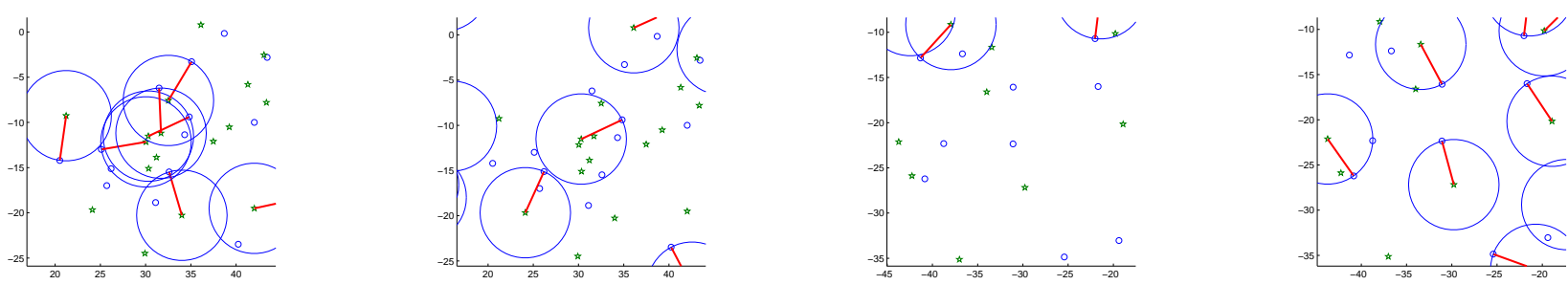

(a) Dense environment using ALOHA. (b) Dense environment using SARA. (c) Sparse environment using ALOHA. (d) Sparse environment using SARA.

Fig. 8. Snapshot of the dense and sparse environment using ALOHA and SARA $\left(\lambda=0.02, \beta=3 \mathrm{~dB}, r_{t}=5 \mathrm{~m}, P=30 \mathrm{dBm}\right)$.
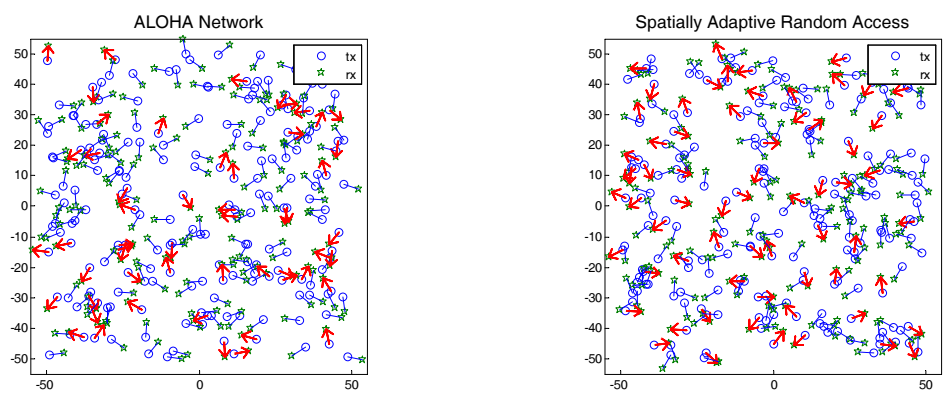

(a) Topology of the active transmitters for the (b) Topology of the active transmitters for SARA. conventional ALOHA.

Fig. 9. Topology of the active transmitters. The active communication pairs are represented as red arrows $\left(\lambda=0.02, \beta=3 \mathrm{~dB}, r_{t}=5 \mathrm{~m}, P=30 \mathrm{dBm}\right)$.

scheme, the active transmitters are overlapped (Figure 9(a)). On the other hand, with SARA, the active transmitters span the entire network (Figure 9(b)). It resembles the topology of the CSMA/CA network.

Figure 10 shows the ASE performance of the various random access schemes. The proposed scheme (SARA) surpasses the conventional ALOHA scheme. In most cases, SARA shows superior performance. The performance difference is severe for the highly dense networks. We conducted the comparison with adaptive ALOHA that is capable of adjusting the transmit probabilities based on locally measured number of nodes. If the number of nodes in the communication area is $N$, the transmit probability is adjusted by $1 / N$ [4]. SARA shows better ASE performance than this scheme for all simulation settings. We also conducted the comparison with two CSMA/CA schemes. The first one is CSMA/CA with fixed sensing range. The carrier sensing range is set by doubling the transmission distance as a conventional setting [34]. The performance of SARA is better than that of the CSMA/CA with fixed sensing range scheme. The second one is CSMA/CA with adaptive sensing range. In this scheme, the receiver initiates the basic carrier sensing range $r_{b}$. The sensing range of CSMA/CA with fixed sensing range could be used for the initial value. The receiver counts its nearby transmitters within its sensing range. If the number of neighbors is $n$, the receiver adjusts the carrier sensing range as $\sqrt[\alpha]{n} r_{b}$, where $\alpha$ is path-loss exponent. In that, if there are many transmitters, then increasing sensing range. Otherwise, if there are a few transmitters, then decreasing sensing range. The sensing range could be decreased until zero in case of $n=0$. By adjusting the carrier sensing range based on the network situation, the performance of CSMA/CA increases. The ASE performance of such adaptive CSMA is slightly better than SARA for all cases. However, to operate adaptive CSMA/CA properly, the receiver should know the number of nearby transmitters. This could be severe burden especially mobile situation.

The random access procedure of current and near future cellular networks is designed based on ALOHA-like random access [35]. SARA is excellent candidate for improving random access performance because applying SARA needs no modification on the protocol. For both CSMA/CA schemes, even though the target SINR threshold increases, the performance of high density situations $(\lambda=0.03-0.06)$ remains almost unchanged. This means that concurrent transmitting nodes are reduced as the target SINR threshold increases. The transmission success probability in CSMA/CA scheme is highly reliable. The increase of data rate $\log _{2}(1+\beta)$ balances decrease of transmitting node density in the area spectral efficiency. On the other hand, SARA accepts the risk of decrease of success probability to increase transmitting node density. For this reason, the performance of SARA is limited with the high target SINR.

\section{Concluding Remarks}

In this paper, we have shown the potential for improvement of the simple random access scheme by utilizing the received SINR. We investigated the performance of the spatial adaptive random access scheme. For the comparison, we analytically derived the optimal transmit probability of the ALOHA scheme in which all transmitters use the same transmit probability. We proposed an adaptive random access scheme in which the transmitters in the network utilize the 


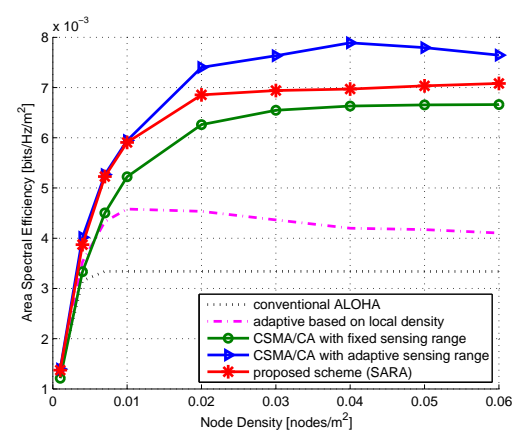

(a) $\beta=3 \mathrm{~dB}$.

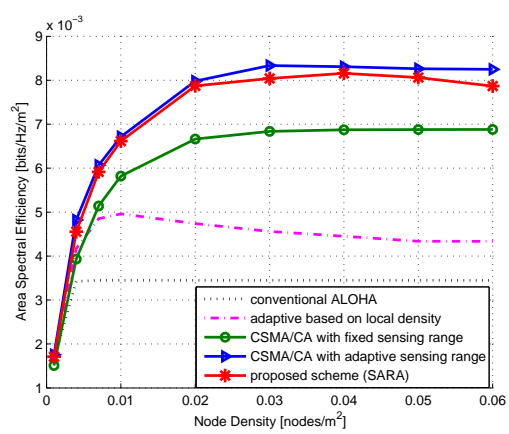

(b) $\beta=5 \mathrm{~dB}$.

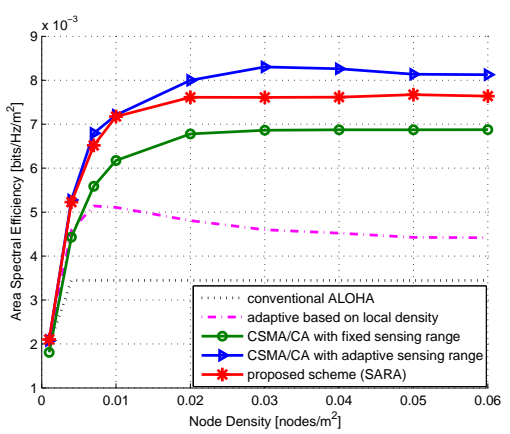

(c) $\beta=7 \mathrm{~dB}$.

Fig. 10. Area spectral efficiency as a function of node density with various $\beta\left(r_{t}=5 \mathrm{~m}, P=30 \mathrm{dBm}\right)$.

different transmit probabilities depending on the situation. The transmit probability is adaptively updated by the ratio of the SINR and the target SINR. We illustrated the performance of the SARA scheme through simulation. We showed that the performance of the spatially adaptive scheme surpasses that of the ALOHA scheme and is comparable with CSMA/CA scheme. The desirable research direction is to design the cost part of utility function (10) considering regional difference. Even though the interferer itself cannot measure its influence to the other nodes in the network without message exchange, if it is possible to reduce the number of communications efficiently, the system performance would more increase.

Additionally, a possible research direction is to find the throughput maximization scheduling under the SINR ratebased interference model, where the instantaneous throughput of transmitter $i, r_{i}$, is the function of the instantaneous SINR at the receiver $k(i)$. That is, the data rate is $r_{i}=\log \left(1+\gamma_{\mathcal{T}_{i}^{\prime}}\right)$. With the adaptive modulation scheme, the data rate is selected according to the channel condition. In this case, the rule for adjusting the transmit probability may differ from that of the SINR-based interference model proposed in this paper.

\section{APPENDIX}

\section{A. Expected value of the number of successfully transmitting nodes}

Transmitters have transmit probabilities, therefore the concurrent transmission nodes are determined stochastically. When transmitter $i$ is transmitting at a given time slot, the probability that the subset $\mathcal{T}_{i}^{j}$ is selected as the concurrent transmission nodes is given by

$$
\begin{aligned}
& \operatorname{Pr}\left[\text { transmitters in } \mathcal{T}_{i}^{j} \text { are active } \mid \text { node } i \text { is active }\right] \\
& =\left(\prod_{l \in \mathcal{T}_{i}^{j}} \phi_{l}\right)\left(\prod_{m \in \mathcal{N} \backslash\left\{\mathcal{T}_{i}^{j}, i\right\}}\left(1-\phi_{m}\right)\right) .
\end{aligned}
$$

The term $\prod_{l \in \mathcal{T}_{i}^{j}} \phi_{l}$ is the probability that all transmitters in set $\mathcal{T}_{i}^{j}$ are transmitting, and the term $\prod_{m \in \mathcal{N} \backslash\left\{\mathcal{T}_{i}^{j}, i\right\}}\left(1-\phi_{m}\right)$ is the probability that all the transmitters, excluding those in $\mathcal{T}_{i}^{j}$ and node $i$, are not transmitting. Using Eq. (22), the average SINR at the receiver of node $i$ can be written as

$$
\Gamma_{k(i)}(\boldsymbol{\Phi})=\phi_{i}\left(\sum_{j=1}^{2^{n-1}}\left(\prod_{l \in \mathcal{T}_{i}^{j}} \phi_{l}\right)\left(\prod_{m \in \mathcal{N} \backslash\left\{\mathcal{T}_{i}^{j}, i\right\}}\left(1-\phi_{m}\right)\right) \gamma_{\mathcal{T}_{i}^{j}}\right),
$$

where $\boldsymbol{\Phi}$ denotes the vector of the transmission probabilities of all transmitters. The conditioned average SINR can be written as

$$
\Gamma_{k(i)}\left(\boldsymbol{\Phi}_{-i} \mid \phi_{i}\right)=\sum_{j=1}^{2^{n-1}}\left(\prod_{l \in \mathcal{T}_{i}^{j}} \phi_{l}\right)\left(\prod_{m \in \mathcal{N} \backslash\left\{\mathcal{T}_{i}^{j}, i\right\}}\left(1-\phi_{m}\right)\right) \gamma_{\mathcal{T}_{i}^{j}},
$$

where $\boldsymbol{\Phi}_{-i}$ denotes the vector of the transmission probabilities of all transmitters except node $i$. Thus, the term $\mathbb{E}\left[\mathbf{1}_{\gamma_{k(i)} \geq \beta}\right]$ is

$$
\mathbb{E}\left[\mathbf{1}_{\gamma_{k(i)} \geq \beta}\right]=\sum_{j=1}^{2^{n-1}}\left(\prod_{l \in \mathcal{T}_{i}^{j}} \phi_{l}\right)\left(\prod_{m \in \mathcal{N} \backslash\left\{\mathcal{T}_{i}^{j}, i\right\}}\left(1-\phi_{m}\right)\right) \mathbf{1}_{\gamma_{\mathcal{T}_{i}^{j}} \geq \beta} .
$$

\section{B. Gradient of utility function}

If gradient is zero, then

$$
\begin{aligned}
\frac{\partial U_{i}\left(\phi_{i}\right)}{\partial \phi_{i}} & =\min \left\{\max \left\{\phi_{\min }, \frac{1}{\beta} g\left(\boldsymbol{\Phi}_{-i}\right)\right\}, \phi_{\max }\right\}-\phi_{i}=0 \\
\phi_{i} & =\min \left\{\max \left\{\phi_{\min }, \frac{1}{\beta} g\left(\boldsymbol{\Phi}_{-i}\right)\right\}, \phi_{\max }\right\},
\end{aligned}
$$

where $g\left(\boldsymbol{\Phi}_{-i}\right)=\left(\sum_{j=1}^{2^{n-1}}\left(\prod_{l \in \mathcal{T}_{i}^{j}} \phi_{l}\right)\left(\prod_{m \in \mathcal{N} \backslash\left\{\mathcal{T}_{i}^{j}, i\right\}}\left(1-\phi_{m}\right)\right) \gamma_{\mathcal{T}_{i}^{j}}\right)$. There are three cases: $\phi_{\min }>g\left(\boldsymbol{\Phi}_{-i}\right) / \beta, \phi_{\min } \leq g\left(\boldsymbol{\Phi}_{-i}\right) / \beta \leq \phi_{\min }$ and $\phi_{\max }<g\left(\boldsymbol{\Phi}_{-i}\right) / \beta$.

1) If $\phi_{\min }>g\left(\boldsymbol{\Phi}_{-i}\right) / \beta$, then $\max \left\{\phi_{\min }, \frac{1}{\beta} g\left(\boldsymbol{\Phi}_{-i}\right)\right\}=\phi_{\min }$. Thus,

$$
\phi_{i}=\min \left\{\phi_{\min }, \phi_{\max }\right\}=\phi_{\min } .
$$

2) If $\phi_{\min } \leq g\left(\boldsymbol{\Phi}_{-i}\right) / \beta \leq \phi_{\min }$, then $\max \left\{\phi_{\min }, \frac{1}{\beta} g\left(\boldsymbol{\Phi}_{-i}\right)\right\}=$ $\frac{1}{\beta} g\left(\boldsymbol{\Phi}_{-i}\right)$. Thus,

$$
\phi_{i}=\min \left\{\frac{1}{\beta} g\left(\boldsymbol{\Phi}_{-i}\right), \phi_{\max }\right\}=\frac{1}{\beta} g\left(\boldsymbol{\Phi}_{-i}\right) .
$$


3) If $\phi_{\max }<g\left(\boldsymbol{\Phi}_{-i}\right) / \beta$, then $\max \left\{\phi_{\min }, \frac{1}{\beta} g\left(\boldsymbol{\Phi}_{-i}\right)\right\}=$ $\frac{1}{\beta} g\left(\boldsymbol{\Phi}_{-i}\right)$. Thus,

$$
\phi_{i}=\min \left\{\frac{1}{\beta} g\left(\boldsymbol{\Phi}_{-i}\right), \phi_{\max }\right\}=\phi_{\max } .
$$

In this way, the point that gradient is zero can represent all solutions including boundaries.

\section{Two-sided scalability of iterative algorithm (12)}

Let

$$
I(\boldsymbol{\Phi})=\frac{1}{\beta}\left(\sum_{j=1}^{2^{n-1}}\left(\prod_{l \in \mathcal{T}_{i}^{j}} \phi_{l}\right)\left(\prod_{m \in \mathcal{N} \backslash\left\{\mathcal{T}_{i}^{j}, i\right\}}\left(1-\phi_{m}\right)\right) \gamma_{\mathcal{T}_{i}^{j}}\right) .
$$

We first denote $\gamma_{\min }$ as $\min \gamma_{\mathcal{T}_{i}^{j}}$. The two-sided scalability has two inequalities. We will prove each inequality as follows.

1) $\forall \theta>1, \frac{1}{\theta} \boldsymbol{\Phi} \leq \boldsymbol{\Phi}^{\prime} \Rightarrow \frac{1}{\theta} I(\boldsymbol{\Phi}) \leq I\left(\boldsymbol{\Phi}^{\prime}\right)$ :

$$
\begin{aligned}
& I\left(\boldsymbol{\Phi}^{\prime}\right)-\frac{1}{\theta} I(\boldsymbol{\Phi})=\frac{1}{\beta}\left(\sum_{j=1}^{2^{n-1}}\left(\prod_{l \in \mathcal{T}_{i}^{j}} \phi_{l}^{\prime}\right)\left(\prod_{m \in \mathcal{N} \backslash\left\{\mathcal{T}_{i}^{j}, i\right\}}\left(1-\phi_{m}^{\prime}\right)\right) \gamma_{\mathcal{T}_{i}^{j}}\right) \\
& -\frac{1}{\theta \beta}\left(\sum_{j=1}^{2^{n-1}}\left(\prod_{l \in \mathcal{T}_{i}^{j}} \phi_{l}\right)\left(\prod_{m \in \mathcal{N} \backslash\left\{\mathcal{T}_{i}^{j}, i\right\}}\left(1-\phi_{m}\right)\right) \gamma_{\mathcal{T}_{i}^{j}}\right) \\
& \geq \frac{\gamma_{\min }}{\beta \theta} \sum_{j=1}^{2^{n-1}}\left\{\left(\prod_{l \in \mathcal{T}_{i}^{j}} \phi_{l}^{\prime}\right)\left(\prod_{m \in \mathcal{N} \backslash\left\{\mathcal{T}_{i}^{j}, i\right\}}\left(1-\phi_{m}^{\prime}\right)\right)-\left(\prod_{l \in \mathcal{T}_{i}^{j}} \phi_{l}\right)\left(\prod_{m \in \mathcal{N} \backslash\left\{\mathcal{T}_{i}^{j}, i\right\}}\left(1-\phi_{m}\right)\right)\right\} \\
& =\frac{\gamma_{\min }}{\beta \theta}(\underbrace{\sum_{j=1}^{2^{n-1}}\left(\prod_{l \in \mathcal{T}_{i}^{j}} \phi_{l}^{\prime}\right)\left(\prod_{m \in \mathcal{N} \backslash\left\{\mathcal{T}_{i}^{j}, i\right\}}\left(1-\phi_{m}^{\prime}\right)\right)}_{=1} \underbrace{\sum_{j=1}^{\sum^{n-1}}\left(\prod_{l \in \mathcal{T}_{i}^{j}} \phi_{l}\right)\left(\prod_{m \in \mathcal{N} \backslash\left\{\mathcal{T}_{i}^{j}, i\right\}}\left(1-\phi_{m}\right)\right)}_{=1}
\end{aligned}
$$$$
=0
$$

$$
\therefore I\left(\mathbf{\Phi}^{\prime}\right) \geq \frac{1}{\theta} I(\boldsymbol{\Phi}) .
$$

2) $\forall \theta>1, \boldsymbol{\Phi}^{\prime} \leq \theta \boldsymbol{\Phi} \Rightarrow I\left(\boldsymbol{\Phi}^{\prime}\right) \leq \theta I(\boldsymbol{\Phi})$ : Using the similar way, we can obtain the following inequality.

$$
\begin{aligned}
& \theta I(\boldsymbol{\Phi})-I\left(\boldsymbol{\Phi}^{\prime}\right) \\
& \geq \frac{\theta \gamma_{\min }}{\beta}(\underbrace{\sum_{j=1}^{2^{n-1}}\left(\prod_{l \in \mathcal{T}_{i}^{j}} \phi_{l}\right)\left(\prod_{m \in \mathcal{N} \backslash\left\{\mathcal{T}_{i}^{j}, i\right\}}\left(1-\phi_{m}\right)\right)}_{=1} \underbrace{\sum_{j=1}^{\sum^{n-1}}\left(\prod_{l \in \mathcal{T}_{i}^{j}} \phi_{l}^{\prime}\right)\left(\prod_{m \in \mathcal{N} \backslash\left\{\mathcal{T}_{i}^{j}, i\right\}}\left(1-\phi_{m}^{\prime}\right)\right)}_{=1} \\
& =0 \\
& \therefore \theta I(\boldsymbol{\Phi}) \geq I\left(\boldsymbol{\Phi}^{\prime}\right) .
\end{aligned}
$$

Finally we get

$$
\frac{1}{\theta} I(\boldsymbol{\Phi}) \leq I\left(\boldsymbol{\Phi}^{\prime}\right) \leq \theta I(\boldsymbol{\Phi}) .
$$

3) $\forall \theta>1, \boldsymbol{\Phi} \leq \theta \boldsymbol{\Phi} \Rightarrow I(\boldsymbol{\Phi}) \leq \theta I(\boldsymbol{\Phi})$ : The value of $\theta I(\boldsymbol{\Phi})-I(\boldsymbol{\Phi})$ is

$$
\begin{gathered}
\left.\theta I(\mathbf{\Phi})-I(\boldsymbol{\Phi})=\frac{1}{\beta}\left(\sum_{j=1}^{2^{n-1}}\left(\prod_{l \in \mathcal{T}_{i}^{j}} \phi_{l}\right)\left(\prod_{m \in \mathcal{N} \backslash\left\{\mathcal{T}_{i}^{j}, i\right\}}\left(1-\phi_{m}\right)\right) \gamma_{\mathcal{T}_{i}^{j}}(\theta-1)\right)\right) \\
\geq 0 \quad \therefore \theta I(\mathbf{\Phi}) \geq I(\boldsymbol{\Phi}) .
\end{gathered}
$$

Finally we get

$$
\frac{1}{\theta} I(\boldsymbol{\Phi}) \leq I(\boldsymbol{\Phi}) \leq \theta I(\boldsymbol{\Phi}) .
$$

\section{Derivation of Eq. (20)}

Point $\phi^{*}$ is a strict local maximizer if it satisfies the following conditions (second order sufficient condition (SOSC)) [36].

1) $\left.\frac{\partial \eta(\phi)}{\partial \phi}\right|_{\phi=\phi^{*}}=0$

The first derivative of $\eta$ is

$$
\frac{\partial \eta}{\partial \phi}=\lambda \log (1+\beta) \exp \left(-\lambda \phi r_{t}^{2} \beta^{2 / \alpha} \rho(\alpha)\right)\left(1-\lambda \phi r_{t}^{2} \beta^{2 / \alpha} \rho(\alpha)\right) .
$$

The value $\hat{\phi}$ that satisfies the first condition is

$$
\hat{\phi}=\frac{1}{\lambda r_{t}^{2} \beta^{2 / \alpha} \rho(\alpha)} .
$$

The second derivative of $\eta$ is

$$
\begin{aligned}
\frac{\partial^{2} \eta}{\partial \phi^{2}} & =\lambda^{2} r_{t}^{2} \beta^{2 / \alpha} \log (1+\beta) \rho(\alpha) \exp \left(-\lambda \phi r_{t}^{2} \beta^{2 / \alpha} \rho(\alpha)\right) \\
& \times\left(-2+\lambda \phi r_{t}^{2} \beta^{2 / \alpha} \rho(\alpha)\right) \\
\left.\frac{\partial^{2} \eta}{\partial \phi^{2}}\right|_{\phi=\hat{\phi}} & =-\lambda^{2} r_{t}^{2} \beta^{2 / \alpha} \log (1+\beta) \rho(\alpha) \exp \left(-\lambda \phi r_{t}^{2} \beta^{2 / \alpha} \rho(\alpha)\right) \\
& <0 .
\end{aligned}
$$

The value $\hat{\phi}$ satisfies the second condition. Since $\hat{\phi}$ satisfies the SOSC, it is a local maximizer and since $\hat{\phi}$ is the only strict local maximizer, it is a global maximizer.

$$
\therefore \phi^{*}=\frac{1}{\lambda r_{t}^{2} \beta^{2 / \alpha} \rho(\alpha)},
$$

where $\rho(\alpha)=\frac{2 \pi^{2}}{\alpha} \csc \left(\frac{2 \pi}{\alpha}\right)$.

\section{REFERENCES}

[1] R. D. Yates, "A framework for uplink power control in cellular radio systems," IEEE Journal on Selected Areas in Communications, vol. 13, no. 7, pp. 1341-1347, September 1995.

[2] C. W. Sung and K.-K. Leung, "A generalized framework for distributed power control in wireless networks," IEEE Transactions on Information Theory, vol. 51, no. 7, pp. 2625-2635, July 2005.

[3] N. Abramson, "THE ALOHA SYSTEM: another alternative for computer communications," in Proc. of the ACM AFIPS 1970, Montvale, New Jersey, November 1970.

[4] D. Bertsekas and R. Gallager, Data Networks, 2nd ed. Prentice-Hall, 1992.

[5] X. Wang and K. Kar, "Distributed algorithms for max-min fair rate allocation in Aloha networks," in Proc. of the Allerton Conference 2004, Monticello, Illinois, USA, September 2004. 
[6] K. Kar, S. Sarkar, and L. Tassiulas, "Achieving proportional fairness using local information in Aloha networks," IEEE Transactions on Automatic Control, vol. 49, no. 10, pp. 1858-1863, October 2004.

[7] X. Wang and K. Kar, "Cross-layer rate control for end-to-end proportional fairness in wireless networks with random access," in Proc. of the ACM MobiHoc 2005, Urbana-Champaign, Illinois, USA, May 2005.

[8] J.-W. Lee, M. Chiang, and A. R. Calderbank, "Utility-optimal randomaccess control," IEEE Transactions on Wireless Communications, vol. 6, no. 7, pp. 2741-2751, July 2007.

[9] A. H. Mohsenian-Rad, J. Huang, M. Chiang, and V. W. S. Wong, "Utility-optimal random access: Reduced complexity, fast convergence, and robust performance," IEEE Transactions on Wireless Communications, vol. 8, no. 2, pp. 898-911, February 2009.

[10] - "Utility-optimal random access without message passing," IEEE Transactions on Wireless Communications, vol. 8, no. 3, pp. 1073-1079, March 2009.

[11] J. Mo and J. Walrand, "Fair end-to-end window-based congestion control," IEEE/ACM Transactions on Networking (ToN), vol. 8, no. 5, pp. 556-567, October 2000.

[12] D. N. C. Tse and P. Viswanath, Fundamentals of Wireless Communication. Cambridge University Press, 2005.

[13] P. Gupta and P. R. Kumar, "The capacity of wireless networks," IEEE Transactions on Information Theory, vol. 46, no. 2, pp. 388-404, March 2000.

[14] D. Stoyan, W. Kendall, and J. Mecke, Stochastic Geometry and its Applications, 2nd ed. Wiley, 1995.

[15] F. Baccelli and B. Błaszczyszyn, Stochastic Geometry and Wireless Networks, Volume I: Theory. Now Publishers Inc, 2009, vol. 1.

[16] _ Stochastic Geometry and Wireless Networks, Volume II: Applications. Now Publishers Inc, 2009, vol. 2.

[17] M. Haenggi and R. K. Ganti, Interference in large wireless networks. Now Publishers Inc, 2001.

[18] M. Haenggi, J. G. Andrews, F. Baccelli, O. Dousse, and M. Franceschetti, "Stochastic geometry and random graphs for the analysis and design of wireless networks," IEEE Journal on Selected Areas in Communications, vol. 27, no. 7, pp. 1029-1046, September 2009.

[19] M. Z. Win, P. C. Pinto, and L. A. Shepp, "A mathematical theory of network interference and its applications," Proceedings of the IEEE, vol. 97, no. 2, pp. 205-230, February 2009.

[20] P. Cardieri, "Modeling interference in wireless ad hoc networks," IEEE Communications Surveys $\mathcal{E}$ Tutorials, vol. 12, no. 4, pp. 551-572, 2010.

[21] J. G. Andrews, R. K. Ganti, M. Haenggi, N. Jindal, and S. Weber, "A primer on spatial modeling and analysis in wireless networks," IEEE Communications Magazine, vol. 48, no. 11, pp. 156-163, November 2010.

[22] S. Weber, J. G. Andrews, and N. Jindal, "An overview of the transmission capacity of wireless networks," IEEE Transactions on Communications, vol. 58, no. 12, pp. 3593-3604, December 2010.

[23] F. Baccelli, B. Błaszczyszyn, and P. Mühlethaler, "An Aloha protocol for multihop mobile wireless networks," IEEE Transactions on Information Theory, vol. 52, no. 2, pp. 421-436, February 2006.

[24] F. Baccelli and C. K. Singh, "Adaptive spatial Aloha, fairness and stochastic geometry," in Proc. of the Workshop on Spatial Stochastic Models for Wireles Networks 2013, Tsukuba Science City, Japan, May 2013.

[25] A.-H. Mohsenian-Rad, V. W. S. Wong, and R. Schober, "Optimal SINRbased random access," in Proc. of the IEEE INFOCOM 2010, San Diego, CA, USA, March 2010.

[26] M. H. Cheung and V. W. S. Wong, "Interference pricing for SINR-based random access game," IEEE Transactions on Wireless Communications, vol. 12, no. 5, pp. 2292-2301, May 2013.

[27] T. Cui, L. Chen, and S. H. Low, "A game-theoretic framework for medium access control," IEEE Journal on Selected Areas in Communications, vol. 26, no. 7, pp. 1116-1127, September 2008.

[28] L. Chen, S. H. Low, and J. C. Doyle, "Random access game and medium access control design," IEEE/ACM Transactions on Networking, vol. 18, no. 4, pp. 1303-1316, August 2010.

[29] M.-S. Alouini and A. J. Goldsmith, "Area spectral efficiency of cellular mobile radio systems," IEEE Transactions on Vehicular Technology, vol. 48, no. 4, pp. 1047-1066, July 1999.

[30] D. M. Kim and S.-L. Kim, "An iterative algorithm for optimal carrier sensing threshold in random CSMA/CA wireless networks," IEEE Communications Letters, vol. 17, no. 11, pp. 2076-2079, November 2013.
[31] S. Weber, X. Yang, J. G. Andrews, and G. de Veciana, "Transmission capacity of wireless ad hoc networks with outage constraints," IEEE Transactions on Information Theory, vol. 51, no. 12, pp. 4091-4102, December 2005.

[32] S. Weber, J. G. Andrews, and N. Jindal, "The effect of fading, channel inversion, and threshold scheduling on ad hoc networks," IEEE Transactions on Information Theory, vol. 53, no. 11, pp. 4127-4149, November 2007.

[33] F. Baccelli, B. Błaszczyszyn, and P. Mühlethaler, "Stochastic analysis of spatial and opportunistic Aloha," IEEE Journal on Selected Areas in Communications, vol. 27, no. 7, pp. 1105-1119, September 2009.

[34] K. Xu, M. Gerla, and S. Bae, "How effective is the IEEE $802.11 \mathrm{rts} / \mathrm{cts}$ handshake in ad hoc networks," in Proc. of the IEEE GLOBECOM 2002 , Taipei, Taiwan, November 2002.

[35] A. Laya, L. Alonso, and J. Alonso-Zarate, "Is the random access channel of lte and lte-a suitable for $\mathrm{m} 2 \mathrm{~m}$ communications? a survey of alternatives," IEEE Communications Surveys $\mathcal{E}$ Tutorials, vol. 16, no. 1, pp. pp. 4-16, 2014.

[36] E. K. P. Chong and S. H. Zak, An introduction to optimization, 2nd ed. Wiley, 2001. 